\title{
Dust Around T Tauri Stars
}

\section{Kyung-Won Suh ${ }^{\dagger}$ and Young-Joo Kwon}

Department of Astronomy and Space Science, Chungbuk National University, Cheongju 361-763, Korea

To reproduce the multiple broad peaks and the fine spectral features in the spectral energy distributions (SEDs) of T Tauri stars, we model dust around T Tauri stars using a radiative transfer model for multiple isothermal circumstellar dust shells. We calculate the radiative transfer model SEDs for multiple dust shells using the opacity functions for various dust grains at different temperatures. For six sample stars, we compare the model results with the observed SEDs including the Spitzer spectral data. We present model parameters for the best fit model SEDs that would be helpful to understand the overall structure of dust envelopes around classical T Tauri stars. We find that at least three separate dust components are required to reproduce the observed SEDs. For all the sample stars, an innermost hot (250-550 K) dust component of amorphous (silicate and carbon) and crystalline (corundum for all objects and forsterite for some objects) grains is needed. Crystalline forsterite grains can reproduce many fine spectral features of the sample stars. We find that crystalline forsterite grains exist in cold regions (80-100 K) as well as in hot inner shells.

Keywords: stars: pre-main sequences, infrared: stars, circumstellar matter, dust: extinction

\section{INTRODUCTION}

T Tauri stars are generally believed to be low-mass $(0.1$ $2 M_{\odot}$ ) pre-main-sequence stars of spectral types $\mathrm{F}$ to $\mathrm{M}$ and surface effective temperatures of 3,000 $\mathrm{K}$ to 7,000 $K$. As a consequence of the star formation process they are surrounded by a gas and dust envelope and/or disk (Bertout 1989). Classical T Tauri stars (CTTS) are optically visible, as they possess a small fraction of their natal envelope. In the spectral energy distribution (SED) classification, CTTS are Class II sources and their SEDs peak between 1 and $10 \mu \mathrm{m}$ with moderate infrared (IR) excesses reaching beyond $100 \mu \mathrm{m}$.

The Spitzer space telescope (Spitzer), launched in 2003, has the infrared spectrograph (IRS) with high sensitivity and large spectral range $(5-35 \mu \mathrm{m})$. The high resolution IRS spectroscopic observations have revealed the detailed SEDs of many T Tauri stars in nearby molecular clouds where various amorphous and crystalline dust features are present (Olofsson et al. 2009). The infrared SEDs of the T Tauri stars show multiple broad peaks at
5-200 $\mu \mathrm{m}$ (Sicilia-Aguilar et al. 2009).

In this paper, we model dust around six sample CTTS using the optical properties of various amorphous and crystalline dust grains at different temperatures. We use a radiative transfer model for multiple isothermal circumstellar dust shells (Towers \& Robinson 2009). Even though the model ignores the disk geometry, it is useful to investigate the overall properties of the multiple dust components around the central star. We compare the model results with the observed SEDs of the stars, including the ground-based, infrared astronomical satellite (IRAS), Spitzer and AKARI data. Using the comparison, we determine the model parameters for the best fit model SEDs that would help us to understand the overall structure of dust envelopes around CTTS.

\section{SAMPLE STARS}

In this paper, we choose six CTTS. For these stars, good quality observational data including the Spitzer IRS spec- (c) This is an Open Access article distributed under the terms of the Creative Commons Attribution Non-Commercial License (http://creativecommons.org/licenses/by-nc/3.0/) which permits unrestricted non-commercial use, distribution, and reproduction in any medium, provided the original work is properly cited.
Received Sep 29, 2011 Revised Oct 31, 2011 Accepted Nov 04, 2011

${ }^{\dagger}$ Corresponding Author

E-mail:kwsuh@chungbuk.ac.kr

Tel: +82-43-261-2315 Fax: +82-43-274-2312 
tral data in a wide wavelength range are available. The sample stars are listed in Table 1.

The high resolution Spitzer IRS spectroscopic observations revealed the detailed SEDs of many T Tauri stars in nearby molecular clouds where various amorphous and crystalline dust features are present (Olofsson et al. 2009). The Spitzer has the infrared array camera (IRAC) designed for observations of faint sources and deep large-area surveys (Fazio et al. 2004). The IRAC has four channels that can obtain broadband images with high sensitivity at 3.6, 4.5, 5.8 and $8.0 \mu \mathrm{m}$ simultaneously.

The AKARI (Murakami et al. 2007) made an all sky survey with the infrared camera (IRC) and far infrared surveyor (FIS). We can use the AKARI point source catalogue (PSC) data at two bands ( 9 and $18 \mu \mathrm{m}$ ) obtained by the IRC, and the bright source catalogue (BSC) data at four bands $(65,90,140$ and $160 \mu \mathrm{m})$ obtained by the FIS. For each object, we have cross-identified the AKARI source by finding the nearest one using the position information supplied the Set of Identifications, Measurements, and Bibliography for Astronomical Data (SIMBAD) database.

For each object, Table 1 lists the IRAS PSC number, the reference for the reduced Spitzer IRS data, the cross-identified AKARI PSC number, the AKARI BSC number, and the cloud name with the distance.

To obtain the standard flux in $\mathrm{W} / \mathrm{m}^{2}$ for all the data, we use the zero-magnitude calibrating method. The zeromagnitude calibrating data are taken from the related references. The observed SEDs of the six stars are displayed in Fig. 1. For IRAS and AKARI catalogue data, we use only good quality $(\mathrm{q}=3)$ data.

\section{DUST ENVELOPE MODEL CALCULATIONS}

The infrared SEDs of the CTTS show multiple broad peaks at 5-200 $\mu \mathrm{m}$ (Sicilia-Aguilar et al. 2009). A reasonable explanation for this would be that there are multiple dust components radiating multiple regions of infrared wavelengths. It is difficult to reproduce the observed SEDs with a single component dust model (e.g., DUSTY code developed by Ivezić \& Elitzur 1997). Many investigators have tried to model the dust around T Tauri stars with a single component dust disk model (Miroshnichenko et al. 1999, Whitney et al. 2003), or using a simple Planck dust radiation law without considering absorption processes (Bouwman et al. 2008, Olofsson et al. 2010).

Suh (2011) modeled dust around Herbig Ae/Be stars using the radiative transfer model for multiple isothermal spherically symmetric circumstellar dust shells developed by Towers \& Robinson (2009), which assumes that each dust shell is in local thermodynamical equilibrium and that the temperature is constant. Dust grains in the innermost shell absorb the radiation from the central star and radiate at the equilibrium temperature. Dust grains in an outer shell absorb the radiation from the central star and the inner shell(s) and radiate at the equilibrium temperature. The scattering of light is ignored. These assumptions would be reasonable approximations for studying dust around $\mathrm{T}$ Tauri stars.

In this paper, we use the radiative transfer model for multiple isothermal circumstellar dust shells (Towers \& Robinson 2009) to reproduce the observed multiple peaks and crystalline dust features. Though it is a relatively simple model, it can use the flexible parameters of dust properties to treat radiative processes through centrally heated multiple dust shells. The code would be useful to investigate the overall properties of complicated distribution of dust around a central star. We model dust envelopes around T Tauri stars using the optical properties of various amorphous and crystalline dust grains at different temperatures.

Molecules in the atmospheres of CTTS make deep absorption features in the near infrared region (Fig. 1). In this paper, we ignore absorption processes by molecules and concentrate on dust around the central star.

Table 1. Sample of classical T Tauri stars.

\begin{tabular}{lclccc}
\hline Name & IRAS PSC & Spitzer IRS (reference) & AKARI PSC & AKARI BSC & Cloud (d [pc]) \\
\hline Haro 1-16 & $16285-2421$ & Olofsson et al. (2009) & $1631334-242737$ & $1631337-242734$ & Oph cloud (187) $^{\mathrm{a}}$ \\
J0843 & $08450-7854$ & Sicilia-Aguilar et al. (2009) & $0843184-790517$ & - & $\eta$ Cha cluster (97) \\
RECX-5 & - & Sicilia-Aguilar et al. (2009) & - & - & $\eta$ Cha cluster (97) \\
RECX-9 & - & Sicilia-Aguilar et al. (2009) & - & - & $\eta$ Cha cluster (97) \\
VW Cha & - & Olofsson et al. (2009) & $1108012-774229$ & $1107526-774234$ & Cha I cloud (165) $^{\mathrm{b}}$ \\
WX Cha & $11085-7720$ & Olofsson et al. (2009) & $1109586-773708$ & - & Cha I cloud (165) $^{\mathrm{c}}$ \\
\hline
\end{tabular}

IRAS: infrared astronomical satellite, PSC: point source catalogue, IRS: infrared spectrograph, BSC: bright source catalogue.

a'From Makarov (2007), bfrom Mamajek et al. (1999), 'from Bertout et al. (1999). 
For the central star, we assume simple blackbody radiation. For each object, we use the best fitting effective temperature and list in Table 2. For all the sample stars, we assume that the luminosity is the same as the solar

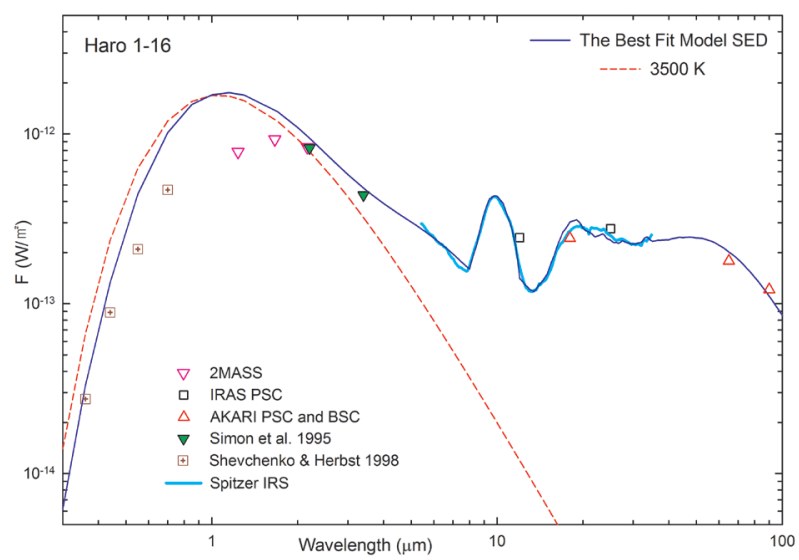

(a)

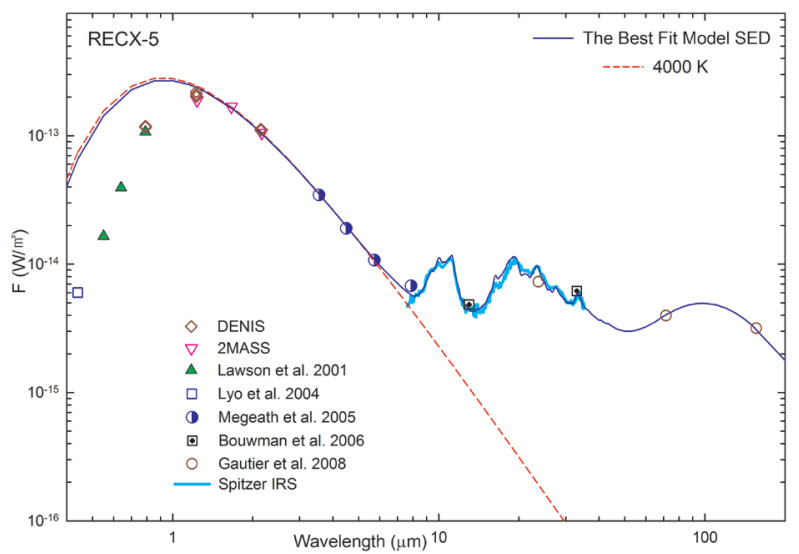

(c)

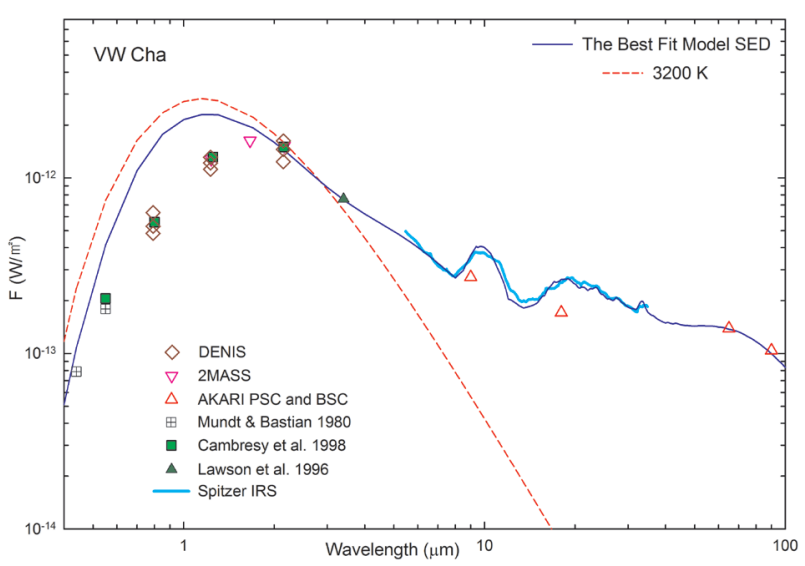

(e) luminosity $\left(L_{*}=1 L L_{\odot}\right)$. A change in the luminosity does not affect the shape of the output SED; it only affects the overall energy output.

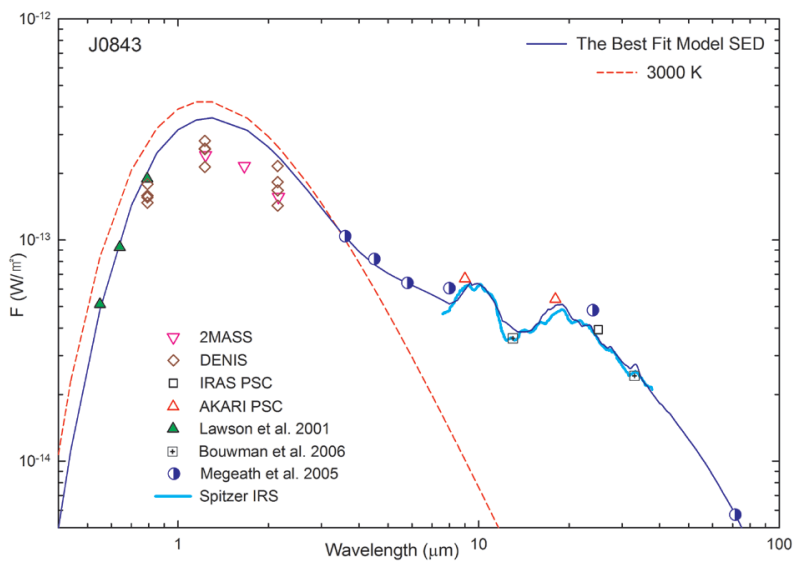

(b)

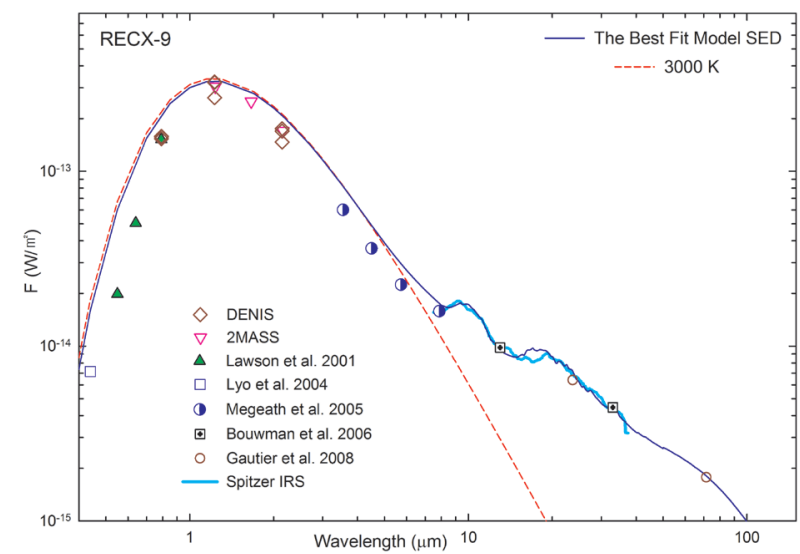

(d)

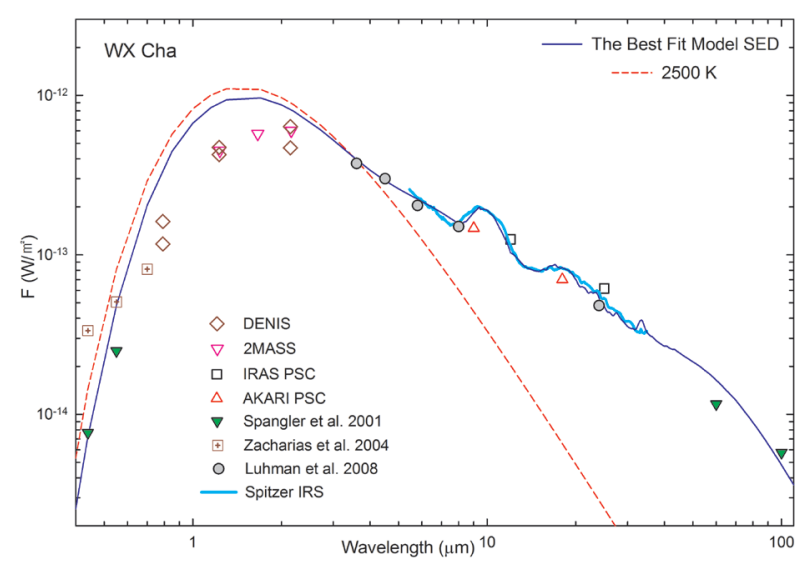

(f)

Fig. 1. Observations compared with model SEDs for the sample stars. SED: spectral energy distribution, IRAS: infrared astronomical satellite, PSC: point source catalogue, IRS: infrared spectrograph. 


\subsection{Dust Opacity}

The best fit model for the observed SED requires a proper combination of multiple isothermal components with different sets of diverse dust opacity functions. We have tried to use as many dust species as possible. We find that four dust species (amorphous silicate, amorphous carbon $[\mathrm{AMC}]$, crystalline corundum and crystalline forsterite) are necessary to reproduce the SEDs of sample stars.

In this paper, we do not consider polycyclic aromatic hydrocarbon for the radiative transfer model calculations, because its thermal properties are not yet well known.

For amorphous silicate, we use the optical constants derived by Suh (1999) for cold silicate. For AMC, we use the optical constants derived by Suh (2000). For the two species, the extinction efficiency factors are calculated for

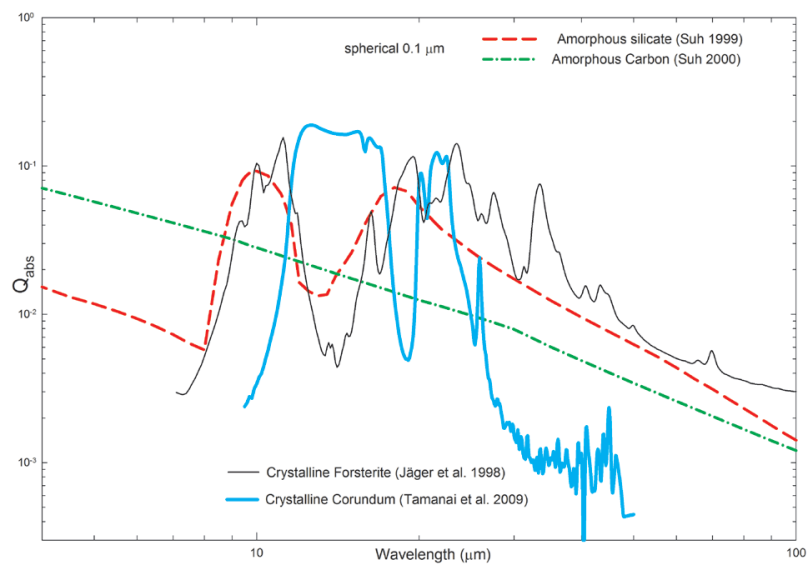

Fig. 2. Dust opacity functions for the four species. spherical dust grains (Bohren \& Huffman 1983) from the optical constants given in the references. The radii of the spherical dust grains are assumed to be $0.1 \mu \mathrm{m}$ uniformly.

For crystalline corundum, we use the extinction data of $\alpha-\mathrm{Al}_{2} \mathrm{O}_{3}$ (corundum-sample 1) obtained by Tamanai et al. (2009). For crystalline forsterite, we use the extinction data obtained by Jäger et al. (1998).

Dust opacity functions for the four species are displayed in Fig. 2. The crystalline forsterite grains produce conspicuous features at 10.0, 11.2, 16.3 19.5, 23.5, 27.5 and $33.5 \mu \mathrm{m}$. We had attempted to use other dust species (olivine, ice and oxides), but found they were not useful for this work.

\subsection{Model SEDs}

We perform various radiative transfer model calculations in the wavelength range 0.01 to $36,000 \mu \mathrm{m}$. We choose $10 \mu \mathrm{m}$ as the fiducial wavelength that sets the scale of the dust optical depth $\left(\tau_{10}\right)$. We have computed the model SEDs for various optical depths of the multiple dust shells with different dust opacity.

For each object, we try to find the best fit model SED for the observations. Once we have a set of reasonable model parameters, we compare the model result with the observed SED and repeatedly revise the related parameter(s) until we get a satisfactory fit in the entire wavelength range. We may have to revise the parameters for the central star and the multiple dust shells repeatedly because of the correlated absorption processes.

The model parameters of the multiple isothermal dust shells for the best fit model SEDs are listed in Table 2. For each object, the parameters of the central star (the black-

Table 2. The model parameters of the multiple isothermal dust shells for the best fit model spectral energy distributions.

\begin{tabular}{lccccc}
\hline \multicolumn{1}{c}{ Name } & $\begin{array}{c}\text { Central star } \\
\mathbf{T}_{\mathrm{BB}}(\mathbf{K})\end{array}$ & $\begin{array}{c}\mathbf{1}^{\text {st }} \text { Shell } \\
\mathbf{T}(\mathbf{K}), \tau_{10}, \mathbf{r}\left(\mathbf{R}_{*}\right)\end{array}$ & Opacity & $\begin{array}{c}2^{\text {nd }} \text { Shell } \\
\mathbf{T}(\mathbf{K}), \tau_{10}, \mathbf{r}\left(\mathbf{R}_{*}\right)\end{array}$ & Opacity $^{1}$ \\
\hline Haro 1-16 & 3,500 & $550,2.4 \mathrm{E}-2,8.8 \mathrm{E} 0$ & $0.3 \mathrm{a}+0.68 \mathrm{~s}+0.02 \mathrm{C}$ & $250,3.0 \mathrm{E}-3,5.3 \mathrm{E} 2$ & $0.6 \mathrm{a}+0.38 \mathrm{~s}+0.02 \mathrm{~F}$ \\
J0843 & 3,000 & $420,9.0 \mathrm{E}-3,1.2 \mathrm{E} 1$ & $0.8 \mathrm{a}+0.17 \mathrm{~s}+0.03 \mathrm{C}$ & $200,4.5 \mathrm{E}-3,7.0 \mathrm{E} 2$ & $0.8 \mathrm{a}+0.18 \mathrm{~s}+0.02 \mathrm{~F}$ \\
RECX-5 & 4,000 & $250,3.2 \mathrm{E}-3,6.8 \mathrm{E} 2$ & $0.4 \mathrm{a}+0.38 \mathrm{~s}+0.02 \mathrm{C}+0.2 \mathrm{~F}$ & $80,1.8 \mathrm{E}-3,1.3 \mathrm{E} 4$ & $0.4 \mathrm{a}+0.58 \mathrm{~s}+0.02 \mathrm{~F}$ \\
RECX-9 & 3,000 & $310,2.3 \mathrm{E}-3,2.6 \mathrm{E} 2$ & $0.77 \mathrm{a}+0.19 \mathrm{~s}+0.02 \mathrm{C}+0.02 \mathrm{~F}$ & $100,9.3 \mathrm{E}-4,4.4 \mathrm{E} 3$ & $0.77 \mathrm{a}+0.19 \mathrm{~s}+0.02 \mathrm{C}+0.02 \mathrm{~F}$ \\
VW Cha & 3,200 & $550,1.5 \mathrm{E}-2,7.5 \mathrm{E} 1$ & $0.6 \mathrm{a}+0.38 \mathrm{~s}+0.02 \mathrm{C}$ & $200,3.0 \mathrm{E}-3,8.2 \mathrm{E} 2$ & $0.8 \mathrm{a}+0.18 \mathrm{~s}+0.02 \mathrm{C}$ \\
WX Cha & 2,500 & $420,1.7 \mathrm{E}-2,7.4 \mathrm{E} 1$ & $0.7 \mathrm{a}+0.27 \mathrm{~s}+0.03 \mathrm{C}$ & $180,1.0 \mathrm{E}-3,5.6 \mathrm{E} 2$ & $0.8 \mathrm{a}+0.15 \mathrm{~s}+0.05 \mathrm{~F}$ \\
\hline \multicolumn{1}{c}{ Name } & - & $3^{\text {rd }}$ Shell & Opacity & $4^{\text {th }}$ Shell & Opacity \\
& - & $\mathbf{T}(\mathbf{K}), \tau_{10}, \mathbf{r}\left(\mathbf{R}_{*}\right)$ & & $\mathbf{T}(\mathbf{K}), \tau_{10}, \mathbf{r}\left(\mathbf{R}_{*}\right)$ & $0.5 \mathrm{a}+0.5 \mathrm{~s}$ \\
Haro 1-16 & - & $100,1.0 \mathrm{E}-2,5.1 \mathrm{E} 3$ & $0.6 \mathrm{a}+0.38 \mathrm{~s}+0.02 \mathrm{~F}$ & $45,1.5 \mathrm{E}-2,4.6 \mathrm{E} 4$ & $0.5 \mathrm{a}+0.5 \mathrm{~s}$ \\
J0843 & - & $100,4.8 \mathrm{E}-3,4.0 \mathrm{E} 3$ & $0.8 \mathrm{a}+0.18 \mathrm{~s}+0.02 \mathrm{~F}$ & $50,2.0 \mathrm{E}-3,2.4 \mathrm{E} 4$ & - \\
RECX-5 & - & $24,2.3 \mathrm{E}-3,4.5 \mathrm{E} 5$ & $0.4 \mathrm{a}+0.6 \mathrm{~s}$ & - & - \\
RECX-9 & - & $40,3.5 \mathrm{E}-4,6.1 \mathrm{E} 4$ & $0.8 \mathrm{a}+0.2 \mathrm{~s}$ & - & $0.5 \mathrm{a}+0.5 \mathrm{~s}$ \\
VW Cha & - & $90,5.0 \mathrm{E}-3,6.4 \mathrm{E} 3$ & $0.8 \mathrm{a}+0.17 \mathrm{~s}+0.03 \mathrm{~F}$ & $35,7.0 \mathrm{E}-3,7.8 \mathrm{E} 4$ & $0.5 \mathrm{a}+0.5 \mathrm{~s}$ \\
WX Cha & - & $100,2.3 \mathrm{E}-3,2.5 \mathrm{E} 3$ & $0.8 \mathrm{a}+0.15 \mathrm{~s}+0.05 \mathrm{~F}$ & $50,2.5 \mathrm{E}-3,1.5 \mathrm{E} 4$ &
\end{tabular}

'a: amorphous carbon (Suh 2000), s: amorphous silicate (Suh 1999), C: crystalline corundum (Tamanai et al. 2009), F: crystalline forsterite (Jäger et al. 1998). 
body temperature) and those for multiple dust shells are listed. For each dust shell, the equilibrium dust temperature, the dust optical depth $\left(\tau_{10}\right)$, the radius of the shell $(\mathrm{r})$ in the unit of the radius of the central star $\left(\mathrm{R}_{t}\right)$ and the dust opacity function are listed. All of the above parameters are input parameters. For each model, the code calculates the radii of the multiple dust shells and the model SED.

\section{SED COMPARISON}

The six panels of Fig. 1 show the best fit model SEDs compared with the observed SEDs for the six sample stars. For all of the sample stars, we use three or four separate dust shells with the four dust species (Table 2). We could reproduce some of the observed fine spectral features with the theoretical models using the crystalline dust grains. Generally, a cold (24-50 K) outer dust shell with amorphous silicate and AMC grains reproduces the FIR region fairly well.

Haro 1-16 shows very conspicuous amorphous silicate features at 10 and $20 \mu \mathrm{m}$ because of abundant (68\%) silicate grains in the innermost shell. The model can reproduce the evident forsterite dust features at 23.5 and 27.5 $\mu \mathrm{m}$ and the vague feature at $33.5 \mu \mathrm{m}$. Crystalline corundum grains in the hot $(550 \mathrm{~K})$ innermost shell improve the fit in the $10-20 \mu \mathrm{m}$ region.

J0843 shows weak crystalline forsterite dust features and unknown features. The model can reproduce the evident forsterite dust features at 23.5, 27.5 and $33.5 \mu \mathrm{m}$.

RECX-5 looks to have the hottest $(4,000 \mathrm{~K})$ central star. The object shows the most conspicuous crystalline forsterite dust features in a wide wavelength range. Of the six sample stars, only this object shows the evident 10.0, 11.2 and $16.3 \mu \mathrm{m}$ features from hot forsterite. The star also shows the conspicuous $23.5,27.5$ and $33.5 \mu \mathrm{m}$ features. All of those features are reproduced by the theoretical model. The content of forsterite in the warm $(250 \mathrm{~K}) \mathrm{in}$ nermost shell is very high (20\%).

RECX-9 shows many weak spectral features in a wide wavelength range. Some of those features are unknown. The model can reproduce the vague forsterite dust features at 23.5, 27.5 and $33.5 \mu \mathrm{m}$. Crystalline corundum grains in inner two shells improve the fit in the $10-30 \mu \mathrm{m}$ region.

VW Cha shows weak crystalline dust features. The crystalline forsterite grains that exist only in the cold (90 K) shell can reproduce the features at 23.5, 27.5 and $33.5 \mu \mathrm{m}$.

WX Cha shows very weak crystalline forsterite features.
The crystalline forsterite grains in the two cold (100 and $180 \mathrm{~K}$ ) shells produce the vague features at 23.5, 27.5 and $33.5 \mu \mathrm{m}$.

For all the sample stars, crystalline corundum in the hot (250-550 K) innermost shell improves the fit in the 10$20 \mu \mathrm{m}$ region.

Even though we have tried to reproduce all the features and characteristics of the observed SEDs by the radiative transfer model, we could not reproduce some of them (e.g., some features in J0843 and RECX-9). There are three possible reasons for this. First, we may not have considered some important dust species. Secondly, the dust opacity functions used for this work may need to be improved. Finally, the radiative transfer model could be too simple. A more sophisticated radiative transfer model, which can consider multiple components of dusty disk and shell with more dust species, would be able to reproduce the SEDs better.

The size of dust grains only affects the overall energy output in the far infrared region. We find that changes of the dust size do not cause meaningful differences in the overall fitting for the sample stars.

\section{DISCUSSION ON CRYSTALLINE DUST}

For all the sample stars, the observed spectral features of crystalline silicate (forsterite) grains are reproduced by the model calculations. Because crystalline silicate grains show very sharp features, even a small content (about $2 \%$ ) can be easily detectable.

Though a small content (2-3\%) of crystalline corundum grains does not reproduce their own sharp spectral features directly, those in the hot (250-550 K) innermost shell can improve the fit in the $10-20 \mu \mathrm{m}$ region for all the sample stars. Similar effects were found for some Herbig Ae/Be stars (Suh 2011).

It is quite evident that crystalline silicate (forsterite) grains exist in cold (48-100 K) outer regions of many $\mathrm{T}$ Tauri stars (this paper, Juhász et al. 2010). Though crystalline silicates are abundant in many young stellar objects (YSOs) and solar system comets, they are essentially missing from the interstellar medium (Juhász et al. 2010). It would be reasonable to assume that crystallization occurs in the low temperature envelopes (or disks) of YSOs.

Some known processes of crystallization (annealing and direct condensation from the gas phase) require high temperature (about 1,000 K) (Fabian et al. 2000). The vertical mixing by turbulence, in disks, can transfer crystalline grain components, which formed in the high 
temperatures and high ultraviolet radiation field of the disk atmosphere, into the disk midplane where the temperature is very low (Dullemond et al. 2006). Olofsson et al. (2009) argued that the abundant crystalline silicates found far from their presumed formation regions (hot regions) may suggest efficient outward radial transport mechanisms in the disks around $\mathrm{T}$ Tauri stars.

On the other hand, Carrez et al. (2002) and Kimura et al. (2008) suggested a mechanism of crystallization at low temperature by reporting that amorphous silicate grains were crystallized to forsterite by electron-beam irradiation. YSOs are known to undergo active and frequent flaring events in which electrons are accelerated. Therefore, the electron irradiation of dust in YSO environments could explain the origin of the crystalline silicate grains around $\mathrm{T}$ Tauri stars.

\section{CONCLUSIONS}

To reproduce the multiple broad peaks and fine spectral features in the SEDs of CTTS, we have modeled dust around CTTS using a radiative transfer model for multiple isothermal circumstellar dust shells. By comparing the model results with the observed SEDs in a wide wavelength range for the six sample stars, we have presented the model parameters of the best fit model SEDs that will be helpful to understand the overall structure of dust envelopes around T Tauri stars.

We have found that at least three separate dust components are required to reproduce the observed SEDs. For all the sample stars, an innermost hot (250-550 K) dust component of amorphous (silicate and carbon) and crystalline (corundum for all objects and forsterite for some objects) grains is needed. We have found that crystalline forsterite grains can reproduce many fine spectral features of the sample stars.

A small content of crystalline corundum grains has been found to be present in all of the sample stars. Corundum appears to be one of the major dust components in YSOs.

For all the sample stars, the crystalline silicate (forsterite) grains exist in cold (80-100 K) outer dust shells as well as in hot inner shells. Even though the reason for the existence of low temperature crystalline silicate is still uncertain, the electron irradiation of dust in YSO environments or a process of outward radial transport could be possible scenarios.

Further investigations with sophisticated theoretical models using more various dust species could reveal use- ful information about the physical and chemical properties of dust around T Tauri stars and the general environments of star forming regions.

\section{ACKNOWLEDGEMENTS}

This work was supported by the research grant of the Chungbuk National University in 2010. This research has made use of the SIMBAD database, operated at CDS, Strasbourg, France. This research is based on observations with AKARI, a JAXA project with the participation of ESA.

\section{REFERENCES}

BertoutC, TTauristars: wild as dust, ARA\&A, 27, 351-395 (1989). http://dx.doi.org/10.1146/annurev.aa.27.090189.002031

Bertout C, Robichon N, Arenou, F, Revisiting Hipparcos data for pre-main sequence stars, A\&A, 352, 574-586 (1999).

Bohren CF, Huffman DR, Absorption and scattering of light by small particles (Wiley, New York, 1983).

Bouwman J, Henning Th, Hillenbrand LA, Meyer MR, Pascucci I, et al., The formation and evolution of planetary systems: grain growth and chemical processing of dust in T Tauri systems, ApJ, 683, 479-498 (2008). http:// dx.doi.org/10.1086/587793

Bouwman J, Lawson WA, Dominik C, Feigelson ED, Henning Th, et al., Binarity as a key factor in protoplanetary disk evolution: Spitzer disk census of the $\eta$ chamaeleontis cluster, ApJ, 653, L57-L60 (2006). http://dx.doi. org/10.1086/510365

Cambrésy L, Copet E, Epchtein N, de Batz B, Borsenberger J, et al., New young stellar object candidates in the Chamaeleon I molecular cloud discovered by DENIS, A\&A, 338, 977-987 (1998).

Carrez P, Demyk K, Leroux H, Cordier P, Jones AP, et al., Lowtemperature crystallization of $\mathrm{MgSiO}_{3}$ glasses under electron irradiation: possible implications for silicate dust evolution in circumstellar environments, M\&PS, 37, 16151622 (2002). http://dx.doi.org/10.1111/j.1945-5100.2002. tb00815.x

Dullemond CP, Apai D, Walch S, Crystalline silicates as a probe of disk formation history, ApJ, 640, L67-L70 (2006). http://dx.doi.org/10.1086/503100

Fabian D, Jäger C, Henning Th, Dorschner J, Mutschke H, Steps toward interstellar silicate mineralogy. V. Thermal evolution of amorphous magnesium silicates and silica, A\&A, 364, 282-292 (2000). 
Fazio GG, Hora JL, Allen LE, Ashby MLN, Barmby P, et al., The infrared array camera (IRAC) for the Spitzer space telescope, ApJS, 154, 10-17 (2004). http://dx.doi. org/10.1086/422843

Gautier TN III, Rebull LM, Stapelfeldt KR, Mainzer A, SpitzerMIPS observations of the $\eta$ Chamaeleontis young association, ApJ, 683, 813-821 (2008). http://dx.doi.org/ 10.1086/589708.

Ivezić A, Elitzur M, Self-similarity and scaling behaviour of infrared emission from radiatively heated dust. I. Theory, MNRAS, 287, 799-811 (1997).

Jäger C, Molster FJ, Dorschner J, Henning Th, Mutschke H, et al., Steps toward interstellar silicate mineralogy. IV. The crystalline revolution, A\&A, 339, 904-916 (1998).

Juhász A, Bouwman J, Henning Th, Acke B, van den Ancker ME, et al., Dust Evolution in Protoplanetary Disks Around Herbig Ae/Be Stars- the Spitzer View, ApJ, 721, 431-455 (2010). http://dx.doi.org/10.1088/0004-637x/ $721 / 1 / 431$

Kimura Y, Miyazaki Y, Kumamoto A, Saito M, Kaito C, Characteristic low-temperature crystallization of amorphous Mg-bearing silicate grains under electron irradiation, ApJ, 680, L89-L92 (2008). http://dx.doi.org/ $10.1086 / 589828$

Lawson WA, Crause LA, Mamajek EE, Feigelson ED, The $\eta$ Chamaeleontis cluster: photometric study of the ROSAT-detected weak-lined T Tauri stars, MNRAS, 321, 5766 (2001). http://dx.doi.org/10.1046/j.1365-8711.2001. 03967.x

Lawson WA, Feigelson ED, Huenemoerder DP, An improved HR diagram for Chamaeleon I pre-main-sequence stars, MNRAS, 280, 1071-1088 (1996).

Luhman KL, Allen LE, Allen PR, Gutermuth RA, Hartmann L, et al., The disk population of the Chamaeleon I starforming region, ApJ, 675, 1375-1406 (2008). http:// dx.doi.org/10.1086/527347

Lyo A, Lawson WA, Bessell MS, The spectroscopic characteristics of intermediate aged pre-main-sequence stars: the $\eta$ Chamaeleontis cluster, MNRAS, 355, 363-373 (2004). http://dx.doi.org/10.1111/j.1365-2966.2004.08318.x

Makarov VV, Signatures of dynamical star formation in the ophiuchus association of pre-main-sequence stars, ApJ, 670, 1225-1233 (2007). http://dx.doi.org/10.1086/522669

Mamajek EE, Lawson WA, Feigelson ED, The $\eta$ Chamaeleontis cluster: a remarkable new nearby young open cluster, ApJ, 516, L77-L80 (1999).

Megeath ST, Hartmann L, Luhman KL, Fazi GG, Spitzer/IRAC photometry of the $\eta$ Chameleontis association, ApJ, 634, L113-L116 (2005). http:/ /dx.doi.org/10.1086/498503

Miroshnichenko A, Ivezić Z, Vinković D, Elitzur M, Dust emission from Herbig Ae/Be stars: evidence for disks and envelopes, ApJ, 520, L115-L118 (1999).

Mundt R, Bastian U, UBV photometry of young emissionline objects, A\&AS, 39, 245-250 (1980).

Murakami H, Baba H, Barthel P, Clements DL, Cohen M, et al., The infrared astronomical mission AKARI, PASJ, 59, S369-S376 (2007).

Olofsson J, Augereau JC, van Dishoeck EF, Merín B, Lahuis F, et al., C2D Spitzer-IRS spectra of disks around T Tauri stars IV. Crystalline silicates, A\&A, 507, 327-345 (2009). http://dx.doi.org/10.1051/0004-6361/200912062

Olofsson J, Augereau JC, van Dishoeck EF, Merín B, Grosso N, et al., C2D Spitzer-IRS spectra of disks around T Tauri stars. V. Spectral decomposition A\&A, 520, A39 (2010). http://dx.doi.org/10.1051/0004-6361/200913909

Shevchenko VS, Herbst W, The search for rotational modulation of T Tauri stars in the ophiuchus dark cloud, AJ, 116, 1419-1431 (1998). http://dx.doi.org/10.1086/300496

Sicilia-Aguilar A, Bouwman J, Juhasz A, Henning Th, Roccatagliata V, et al., The long-lived disks in the $\eta$ Chamaeleontis cluster, ApJ, 701, 1188-1203 (2009). http:// dx.doi. org/10.1088/0004-637x/701/2/1188

Simon M, Ghez AM, Leinert Ch, Cassar L, Chen WP, et al., A lunar occultation and direct imaging survey of multiplicity in the Ophiuchus and Taurus starforming regions, ApJ, 443, 625-637 (1995). http://dx.doi.org/10.1086/175554

Spangler C, Sargent AI, Silverstone MD, Becklin EE, Zuckerma B, Dusty debris around solar-type stars: temporal disk evolution, ApJ, 555, 932-944 (2001). http://dx.doi. org/10.1086/321490

Suh KW, Dust around Herbig AE/Be stars, JKAS, 44, 13-21 (2011). http://dx.doi.org/10.5303/jkas.2011.44.1.13

Suh KW, Optical properties of the carbon dust grains in the envelopes around AGB stars, MNRAS, 315, 740-750 (2000). http://dx.doi.org/10.1046/j.1365-8711.2000.03482.x

Suh KW, Optical properties of the silicate dust grains in the envelopes around AGB stars, MNRAS, 304, 389-405 (1999). http://dx.doi.org/10.1046/j.1365-8711.1999.02317.x

Tamanai A, Mutschke H, Blum J, Posch Th, Koike C, et al., Morphological effects on IR band profiles. Experimental spectroscopic analysis with application to observed spectra of oxygen-rich AGB stars, A\&A, 501, 251-267 (2009). http://dx.doi.org/10.1051/0004-6361/200911614

Towers IN, Robinson G, A model for multiple isothermal circumstellar dust shells, PhyS, 80, 015901 (2009). http:// dx.doi.org/10.1088/0031-8949/80/01/015901

Whitney BA, Wood K, Bjorkman JE, Wolff MJ, Two-dimensional radiative transfer in protostellar envelopes. I. Effects of geometry on Class I sources, ApJ, 591, 1049-1063 (2003). http://dx.doi.org/10.1086/375415 
Zacharias N, Monet DG, Levine SE, Urban SE, Gaume R, et al., The Naval Observatory Merged Astrometric Dataset (NOMAD), in American Astronomical Society 205th Meeting, San Diego, CA, 9-13 Jan 2004, \#48.15. 